\title{
Optimización de la formulación de tallarines libres de gluten enriquecidos con pulpa de zapallo deshidratada empleando el método de diseño de mezclas
}

\section{Optimization of the formulation of gluten-free pasta enriched with dehydrated pumpkin pulp using the method of mix design}

\author{
Natali López-Mejía ${ }^{1 *}$ (D), Nelly Bibiana Morales Posada ${ }^{1}$ \\ ${ }^{1}$ Universitaria Agustiniana, Facultad de Arte, Comunicación y Cultura, Tecnología en Gastronomía, Bogotá D.C. - \\ Colombia \\ *Corresponding Author: Natali López-Mejía, Universitaria Agustiniana, Facultad de Arte, Comunicación y Cultura, \\ Tecnología en Gastronomía, Ak. 86 \#11b-95, 110811, Bogotá D.C. - Colombia, e-mail: \\ natali.lopez@uniagustiniana.edu.co, nalopezme@gmail.com
}

Cite as: López-Mejía, N., \& Morales Posada, N. B. (2020). Optimización de la formulación de tallarines libres de gluten enriquecidos con pulpa de zapallo deshidratada empleando el método de diseño de mezclas. Brazilian Journal of Food Technology, 23, e2018299. https://doi.org/10.1590/1981-6723.29918

\begin{abstract}
Resumen
La pulpa de zapallo deshidratada (Cucurbita máxima) ha sido empleada para enriquecer pastas libres de gluten, debido a su alto contenido nutricional; sin embargo, no se han llevado a cabo estudios donde se logre identificar la mejor formulación a partir de diseños experimentales de optimización. Por lo tanto, el objetivo principal de este trabajo fue evaluar la calidad de los tallarines libres de gluten de cada uno tratamientos desarrollados y utilizar el diseño de mezclas simplex para la obtención de la formulación óptima. Para ello, además de pulpa de zapallo deshidratada dos materias primas fueron consideradas: harina de arroz y harina de maíz. Como variables de respuesta se contemplaron: análisis proximal (contenido de proteína, almidón, cenizas y fibra dietaria), esfuerzo a la fractura, calidad de cocción, color

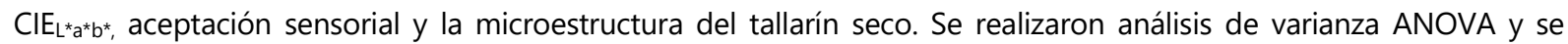
obtuvieron los modelos de regresión (lineal, cuadrático, cúbico especial y cúbico completo), los cuales se emplearon para explicar cada una de las variables de respuesta mencionadas, siendo las de mayor significancia y mayor $R^{2}$ y $R^{2}$ ajustados la adhesividad sensorial, masticabilidad sensorial y ganancia de peso. Finalmente, la formulación que presentó valores óptimos en dichas variables fue aquella con $0,10 \mathrm{~g}$ de pulpa de zapallo deshidratada/g harina; 0,3859 g de harina de arroz/g harina y 0,5141 g de harina de maíz/g harina (valores predichos).
\end{abstract}

Palabras clave: Cucurbita máxima; Harinas libres de gluten; Regresión; Adhesividad; Masticabilidad; Ganancia de peso.

\section{Abstract}

Dehydrated pumpkin pulp (Cucurbita maxima) has been used to enrich gluten-free pasta due to its high nutritional content. However, there is a need for studies to identify the best formulation based on experimental optimization designs. Therefore, this work aimed to evaluate the quality of the gluten-free noodles of each developed treatment 
and use the design of simplex mixtures to obtain the optimal formulation. For this, in addition to dehydrated pumpkin pulp, we considered two other raw materials, rice flour and cornmeal. Response variables included proximal analysis (protein content, starch, ashes and dietary fiber), fracture stress, cooking quality, $\mathrm{ClE}_{\mathrm{L}^{*} \mathrm{a}^{*} \mathrm{~b}^{*}} \mathrm{Color}_{\text {, }}$ sensory acceptance and microstructure of dry noodles. We performed ANOVA analysis of variance and regression models (linear, quadratic, special cubic and complete cubic), which were used to explain each of the response variables, the most significant and highest being $R^{2}$ and $R^{2}$ Adjusted sensory adhesiveness, sensory chewiness and weight gain. Finally, the formulation that presented optimal values in these variables was $0.10 \mathrm{~g}$ of dehydrated pumpkin pulp/g flour; $0.3859 \mathrm{~g}$ of rice flour $/ \mathrm{g}$ flour and $0.5141 \mathrm{~g}$ of cornmeal $/ \mathrm{g}$ flour (predicted values).

Keywords: Cucurbita maxima; Gluten-free flours; Regression; Adhesiveness; Chewiness; Weight gain.

\section{Introducción}

Cada vez es mayor la tendencia al desarrollo de productos alimenticios libres de gluten, debido a la enfermedad celíaca, la cual afecta 1 de cada 100 personas en el mundo provocando inflamación crónica del intestino delgado, dañando la mucosa intestinal y causando la mala absorción de nutrientes (Flores-Silva et al., 2014). También existe intolerancia al gluten no celiaca cuyos síntomas y tratamientos son similares, con una prevalencia en la población 10 veces mayor (13\% de la población) (Catassi et al., 2013), siendo el único tratamiento eficaz es la adherencia de por vida a una dieta libre de gluten (LG) (Catassi et al., 2015).

Por otro lado, cada vez es mayor la preocupación de los consumidores por alimentarse con productos que le proporcionen los nutrientes necesarios para prevenir enfermedades nutricionales; de esta forma buscan mejorar su bienestar físico y mental. Es por esto, que se ha observado una tendencia en el desarrollo de pastas alimenticias sin gluten fortificadas con fuentes de alto valor proteico y vitamínico; sin embargo, aún significa un reto debido a que el gluten es el principal componente que le confiere a la pasta la elasticidad y baja adherencia característica, y al ser reemplazado dichas características se ven directamente afectadas (Mastromatteo et al., 2012).

Una de las harinas vegetales empleadas para enriquecer nutricionalmente pastas alimenticias artesanales es la pulpa de zapallo deshidratada, debido a su alto contenido de fibra dietaria $(34,12 \mathrm{~g} / 100 \mathrm{~g})$, proteína (15,08 g/100 g), carotenoides totales $(511,16 \pm 2,97 \mu \mathrm{g} / \mathrm{g})$, compuestos fenólicos y alta actividad antioxidante (López-Mejía et al., 2019). Diversas investigaciones se han desarrollado con el objetivo evaluar el efecto de la adición de PZD en 3-4 niveles (5-50\%) entre las características más importantes se define la calidad de las pastas y la mejor formulación. De manera general, se ha encontrado que la incorporación de zapallo en pastas frescas mejora el color, afecta negativamente el sabor y la textura de las pastas alimenticias; así como también aumenta el tiempo de cocción y la ganancia de peso. Mirhosseini et al. (2015) evidenciaron que de las 2 formulaciones desarrolladas (25 y 50\%) la de $25 \%$ presentó mejores características de calidad. Posteriormente, Minarovičová et al. (2017) formularon pastas alimenticias con 5; 7,5 y 10\% PZD, encontrando que la formulación con $10 \%$ PZD presenta mayor aceptación sensorial. Finalmente, en el estudio de López-Mejía et al. (2019) se formularon pastas alimenticias con 2 niveles de incorporación de PZD (5 y 10\%) encontrando que la formulación con 10\% PZD muestra la mejor calidad nutricional. Sin embargo, de las investigaciones mencionadas, solo la desarrollada por Mirhosseini et al. (2015) ha sido para pastas libres de gluten.

Se evidencia que hasta la fecha los diseños experimentales incorporan pocos niveles de adición de PZD y solo permiten la variación de un componente, limitando así la selección de una formulación óptima. Por lo anterior, se requiere el empleo de diseños experimentales de optimización como superficie de respuesta o diseño de mezclas (Bastos et al., 2016), siendo este último el adecuado cuando se requiere la variación de 3 componentes simultáneamente. Según Marti et al. (2018), Marengo et al. (2018) y Camelo-Méndez et al. (2018) la harina de arroz y de maíz son ampliamente utilizadas en la formulación de pastas alimenticias, por 
lo que se buscó hallar una formulación óptima de pasta alimenticia seca, tipo tallarín, libre de gluten (TLG) formulada con harina de arroz (HA) y harina de maíz (HM), enriquecida con pulpa de zapallo deshidratada (PZD), con características tecnológicas, fisicoquímicas y sensoriales aceptables, empleando el diseño de mezclas simplex de 10 niveles (incluyendo el control), con análisis de varianza en términos lineal, cuadrático, cúbico completo y cúbico especial para determinar el ajuste en variables como adhesividad sensorial, masticabilidad sensorial y ganancia de peso. Adicionalmente, se buscó evaluar el efecto de cada tratamiento en la calidad de TLG (análisis proximal, esfuerzo a la fractura, calidad de cocción, color, microestructura y aceptabilidad sensorial).

\section{Materiales y métodos}

\subsection{Materia prima}

Zapallo (Cucurbita máxima) de la variedad Boyacá, se obtuvo de un cultivo local del municipio Boyacá (Cundinamarca - Colombia). Demás materias primas mencionadas en este estudio fueron obtenidas de un mercado local. La pulpa de zapallo deshidratada (PZD) se elaboró siguiendo el método reportado por López-Mejía et al. (2019).

El zapallo se seleccionó de acuerdo con el color (color naranja en toda la superficie), descartando aquellas que mostraban signos de deterioro. Posteriormente, se lavó para eliminar el material extraño y luego se desinfectó con ácido peracético $(3 \mathrm{~mL} / \mathrm{L})$. Cada fruto se dividió en ocho partes similares, se retiró la semilla y se escaldó $\left(60{ }^{\circ} \mathrm{C} / 10 \mathrm{~min}\right)$. Las frutas se enfriaron hasta $25 \pm 1{ }^{\circ} \mathrm{C}$, y la cáscara se separó de la pulpa. El mesocarpio se procesó en formas geométricas rectangulares $(3,00 \times 3,01 \times 0,38 \mathrm{~cm})$ y luego se secó en un horno convectivo (RATIONAL, SCC WE $61 \mathrm{G}$, Alemania) a $55^{\circ} \mathrm{C}$ por $24 \mathrm{~h}$ con aire seco $(1,5 \mathrm{~m} / \mathrm{s})$. Una vez seco, la pulpa deshidratada se molió y se tamizó hasta obtener un tamaño de partícula menor a $212 \mu \mathrm{m}$. Posteriormente, la pulpa de zapallo deshidratada (PZD) fue guardada en frascos de polietileno de alta densidad color ámbar bajo refrigeración.

\subsection{Tallarines: formulación y procesamiento}

Además de los componentes experimentales (Pulpa de Zapallo Deshidratada - PZD, Harina de Arroz - HA y Harina de Maíz - HM) (62,5 g de mezcla de harinas/100 g) en las proporciones indicadas en la Tabla 1, se adicionó sal ( $1 \mathrm{~g} / 100 \mathrm{~g})$, aceite de semillas de girasol (3 g/100 g), y huevo (33,5 g/100 g) (Fiorda et al., 2013; Mirhosseini et al., 2015). Los componentes se mezclaron en un homogeneizador por 15 min (Fiorda et al., 2013). Las mezclas se amasaron manualmente y se moldearon en una máquina manual para hacer pasta (IMPERIA, Italia) en forma de tallarín. La pasta se secó en el mismo horno citado para la deshidratación de la pulpa de zapallo, con circulación de aire a $50{ }^{\circ} \mathrm{C} / 70 \% \mathrm{HR}$ por $12 \mathrm{~h}$.

Tabla 1. Diseño experimental para la optimización de la formulación de tallarines libres de gluten enriquecidos con pulpa de zapallo deshidratada (Cucurbita máxima).

\begin{tabular}{|c|c|c|c|c|c|c|}
\hline \multirow{2}{*}{$\begin{array}{c}\text { Orden de } \\
\text { experimentación }\end{array}$} & \multirow{2}{*}{$\begin{array}{l}\text { Número de } \\
\text { corridas }\end{array}$} & \multirow{2}{*}{ Bloques } & \multirow{2}{*}{$\begin{array}{l}\text { Tipo de } \\
\text { punto }\end{array}$} & \multicolumn{3}{|c|}{ Proporción } \\
\hline & & & & $\mathbf{X}_{1}$ & $\mathbf{X}_{2}$ & $\mathbf{X}_{3}$ \\
\hline 4 & 1 & 1 & 1 & 0,1000 & 0,30000 & 0,60000 \\
\hline 9 & 2 & 1 & -1 & 0,1375 & 0,30625 & 0,55625 \\
\hline 8 & 3 & 1 & -1 & 0,2125 & 0,35625 & 0,43125 \\
\hline 3 & 4 & 1 & 1 & 0,2500 & 0,40000 & 0,35000 \\
\hline 7 & 5 & 1 & -1 & 0,1375 & 0,35625 & 0,50625 \\
\hline 2 & 6 & 1 & 1 & 0,1000 & 0,40000 & 0,50000 \\
\hline 6 & 7 & 1 & -1 & 0,2125 & 0,23125 & 0,55625 \\
\hline 1 & 8 & 1 & 1 & 0,2500 & 0,15000 & 0,60000 \\
\hline 5 & 9 & 1 & 0 & 0,1750 & 0,31250 & 0,51250 \\
\hline
\end{tabular}

$\mathrm{X}_{1}$ : Pulpa de Zapallo Deshidratada (g/100 g harina); $\mathrm{X}_{2}$ : Harina de Arroz (g/100 g harina); $\mathrm{X}_{3}$ : Harina de Maíz (g/100 g harina). 


\subsection{Análisis proximal}

El análisis proximal consistió en la cuantificación de humedad (Gravimetría), proteína (International Organization for Standardization, 2009), fibra dietaria (Association of Official Analytical Chemists, 2003), grasa (Instituto Colombiano de Normas Técnicas y Certificación, 1973), cenizas (American Association of Cereal Chemists, 2001) y almidón (Instituto Colombiano de Normas Técnicas y Certificación, 2009) de PZD, HA, HM y de cada uno de los tallarines secos formulados. El análisis se llevó a cabo por triplicado por un laboratorio certificado (ENZIPAN, Bogotá D.C., Colombia).

\subsection{Esfuerzo a la fractura y calidad de cocción}

La resistencia a la fractura $(\mathrm{N})$ fue medida 5 veces en cada uno de los tratamientos evaluados, a partir del cual se calculó el esfuerzo a la fractura $\left(\mathrm{N} / \mathrm{mm}^{2}\right)$. Este análisis fue realizado por un laboratorio externo certificado (Laboratorios M\&G S.A.S, Bogotá D.C, Colombia). La geometría de las muestras fue rectangular $\mathrm{y}$ las dimensiones en promedio tuvieron un espesor $=1,51 \pm 0,07 \mathrm{y}$ un ancho $=8,18 \pm 0,57 \mathrm{~mm}$.

Por otro lado, los ensayos de cocción se realizaron por triplicado para cada formulación de TLG de acuerdo al método AACC 66-50 (American Association of Cereal Chemists, 2000) con algunas modificaciones. Estos consistieron en la inmersión de $25 \mathrm{~g}$ de pasta seca en $300 \mathrm{~mL}$ de agua destilada a $98^{\circ} \mathrm{C}$. El tiempo de cocción se medió como el instante en el que el espagueti alcanza el punto al dente. La ganancia de peso (agua absorbida por la estructura) se determinó por diferencia de peso antes y después de la cocción y se reportó como g de agua/100 g muestra. Finalmente, los sólidos perdidos (SP) por la cocción se evaluaron después de la cocción y el nivel de agua se llevó a volumen inicial. La materia seca se determinó a partir de una muestra de $25 \mathrm{~mL}$ de agua de la cocción sometida a $105^{\circ} \mathrm{C}$ hasta peso constante en un horno. El resultado se expresó como $\mathrm{g}$ de sólidos/100 $\mathrm{g}$ de muestra.

\subsection{Color}

Las coordenadas de color $\mathrm{CIE}_{\mathrm{L}^{*} \mathrm{a}^{*} \mathrm{~b}^{*}}$ fueron determinadas 5 veces en TLG de cada tratamiento, por un laboratorio certificado (Laboratorios M\&G S.A.S, Bogotá D.C, Colombia). Los parámetros que se usaron en la determinación fueron iluminante D 65 y ángulo del observador $10^{\circ}$ con base en la norma internacional ASTM D2244-16 (American Society for Testing and Materials, 2016). El color se medió en términos de coordenadas $\mathrm{L}^{*}$ (luminosidad), a* (color rojizo $(+)$ y verde $(-)$ ) y b* (color amarilloso $(+)$ y azuloso $(-)$ ) (Mendoza et al., 2006).

\subsection{Microestructura}

Las micrografías correspondientes a la sección transversal de los tratamientos con mayor proporción de PZD $(0,25)$ y menor proporción de PZD $(0,10)$ fueron obtenidas en la Universidad de Los Andes (Bogotá D.C., Colombia) empleando un Microscopio Electrónico de Barrido (MEB) (JEOL, JSM 6490-LV) donde se aplicó una tensión de aceleración de $20 \mathrm{kV}$. Una pieza de tallarín seco, unida a un disco de carbón con una cinta plateada se roció con oro en el sublimador de vacío para observar la sección transversal con diferentes aumentos $(30 \mathrm{x}, 200 \mathrm{x}$ y $800 \mathrm{x})$.

\subsection{Análisis sensorial}

Se llevaron a cabo análisis sensoriales de aceptación en tallarines cocidos de los tratamientos evaluados. Cada análisis se realizó con un panel no entrenado de 30 personas (muestra no probabilística por conveniencia) en un lugar iluminado, libre de ruido, olores y colores extraños. Se evaluaron atributos como color, olor, elasticidad, adhesividad, masticabilidad y sabor. Para ello, se empleó una escala hedónica de 
5 puntos, donde 1 correspondió a me disgusta mucho, 2 a me disgusta moderadamente, 3 a no me gusta ni me disgusta, 4 a me gusta moderadamente y 5 a me gusta mucho (Carpenter et al., 2009).

\subsection{Diseño experimental y análisis estadístico}

Para encontrar la formulación óptima de tallarín libre de gluten enriquecido con PZD se empleó un diseño de mezclas simplex (Tabla 1). Los límites máximos y mínimos de cada una de las harinas se fijaron de acuerdo con resultados reportados en la literatura (PZD-bajo: 0,10; PZD-máximo: 0,25; HA-bajo: 0,15; HA-máximo: 0,40; HM-bajo: 0,35 y HM-máximo: 0,60). Las variables de respuesta (aceptación sensorial del color, olor, adhesividad, elasticidad, masticabilidad y sabor; composición proximal, esfuerzo a la fractura, calidad de cocción), fueron seleccionadas de acuerdo con los valores de $\mathrm{R}^{2}$ experimental y ajustado $(>0,9)$ y significancia $(p<0,05)$. A partir de lo anterior, se desarrollaron cuatro modelos matemáticos que las describen (lineal, cuadrático, cúbico especial y cúbico completo).

Por otro lado, para evaluar el efecto de la adición de PZD en las características fisicoquímicas, calidad de cocción, esfuerzo a la fractura y aceptación sensorial se llevó a cabo un análisis de varianza ANOVA con prueba de Tukey y se identificaron diferencias significativas $(p<0,05)$. Adicionalmente se hicieron correlaciones de Pearson para encontrar la relación entre los valores de esfuerzo a la fractura y proporción de PZD en las mezclas, el tamaño del poro y el esfuerzo de fractura, así como el tamaño del poro con la proporción de PZD en la mezcla. Todos los análisis estadísticos de este estudio se realizaron en Minitab v.17.

\section{Resultados y discusión}

\subsection{Análisis proximal}

La Tabla 2 muestra algunos parámetros de la composición química de las materias primas y de los tallarines libres de gluten. PZD se destacó por su alto contenido de proteína $(9,31 \pm 0,05 \mathrm{~g} / 100 \mathrm{~g})$, bajo contenido de grasa $(2,18 \pm 0,02 \mathrm{~g} / 100 \mathrm{~g})$, alto contenido de fibra dietaria $(30,51 \pm 0,02 \mathrm{~g} / 100 \mathrm{~g})$ y cenizas $(10,95 \pm 0,01 \mathrm{~g} / 100 \mathrm{~g})$, respecto a lo evidenciado para HA y HM. Al comparar los valores obtenidos con lo reportado para PZD de otros estudios, se evidencia que Minarovičová et al. (2017) presentaron menores valores para proteína $(8,2 \pm 0,1 \mathrm{~g} / 100 \mathrm{~g})$, grasa $(0,7 \pm 0,0 \mathrm{~g} / 100 \mathrm{~g})$ y cenizas $(2,3 \pm 0,0 \mathrm{~g} / 100 \mathrm{~g})$ y valores similares para fibra dietaria $(27,4 \pm 0,7 \mathrm{~g} / 100 \mathrm{~g})$. Usha Junior et al. (2010) reportan valores más altos de proteína (15,69 g/100 g) y Escalada Plaa et al. (2007) valores más altos de fibra dietaria (44,6 g/100 g). Estas diferencias se deben a características agronómicas del cultivo y a la variedad empleada.

Teniendo en cuenta lo evidenciado para PZD en cuanto a su alto contenido de fibra dietaria, proteína y cenizas, el aumento de la calidad nutricional de TLG se debe a la incorporación de PZD en la formulación, más que a la adición de los demás componentes, razón principal por la que se emplea PZD en pastas alimenticias libres de gluten (PLG). Similar a lo obtenido en este estudio, Mirhosseini et al. (2015) evidenciaron que al incorporar la pulpa de zapallo deshidratada (50\%) se afectaba positivamente el contenido de cenizas - minerales - (no midieron contenido de proteína y fibra dietaria); sin embargo, el contenido de cenizas reportada por los autores para PLG con 25\% PZD $(1,15 \mathrm{~g} / 100 \mathrm{~g})$, es menor a la obtenida en este estudio para PLG con 25\% PZD (3,31-3,57 g/100 g); debido a que la pulpa de zapallo deshidratada que emplean dichos autores, tiene un menor contenido de cenizas $(6 \mathrm{~g} / 100 \mathrm{~g})$, respecto a la de este estudio. 
Tabla 2. Composición proximal de las materias primas y de las muestras de tallarines libres de gluten enriquecidos con pulpa de zapallo deshidratada.

\begin{tabular}{cccccc}
\hline $\mathbf{T}$ & $\begin{array}{c}\text { Proteína } \\
\mathbf{( g / 1 0 0} \mathbf{g})\end{array}$ & $\begin{array}{c}\text { Grasa } \\
\mathbf{( g / 1 0 0} \mathbf{g})\end{array}$ & $\begin{array}{c}\text { Cenizas } \\
(\mathbf{g} / \mathbf{1 0 0} \mathbf{g})\end{array}$ & $\begin{array}{c}\text { Fibra dietaria } \\
\mathbf{( g / 1 0 0} \mathbf{g})\end{array}$ & $\begin{array}{c}\text { Almidón } \\
(\mathbf{g} / \mathbf{1 0 0} \mathbf{g})\end{array}$ \\
\hline HA & $7,41 \pm 0,02$ & $0,43 \pm 0,02$ & $0,43 \pm 0,026$ & $1,22 \pm 0,03$ & $88,45 \pm 0,02$ \\
\hline HM & $8,10 \pm 0,03$ & $4,21 \pm 0,01$ & $1,39 \pm 0,02$ & $11,18 \pm 0,015$ & $66,78 \pm 0,01$ \\
\hline PZD & $9,31 \pm 0,05$ & $2,18 \pm 0,02$ & $10,95 \pm 0,01$ & $30,51 \pm 0,02$ & $0,03 \pm 0,02$ \\
\hline T1 & $9,92 \pm 0,01^{\mathrm{a}}$ & $4,84 \pm 0,01^{\mathrm{a}}$ & $3,57 \pm 0,01^{\mathrm{a}}$ & $13,73 \pm 0,01^{\mathrm{a}}$ & $50,86 \pm 0,00^{\mathrm{i}}$ \\
\hline T2 & $9,59 \pm 0,01^{\mathrm{g}}$ & $4,26 \pm 0,01^{\mathrm{g}}$ & $1,94 \pm 0,00^{\mathrm{i}}$ & $8,42 \pm 0,01^{\mathrm{i}}$ & $65,59 \pm 0,00^{\mathrm{a}}$ \\
\hline T3 & $9,78 \pm 0,01^{\mathrm{c}}$ & $4,00 \pm 0,00^{\mathrm{i}}$ & $3,31 \pm 0,00^{\mathrm{b}}$ & $11,20 \pm 0,01^{\mathrm{c}}$ & $56,05 \pm 0,00^{\mathrm{g}}$ \\
\hline T4 & $9,64 \pm 0,00^{\mathrm{f}}$ & $4,59 \pm 0,01^{\mathrm{c}}$ & $2,04 \pm 0,00^{\mathrm{h}}$ & $9,44 \pm 0,02^{\mathrm{h}}$ & $63,52 \pm 0,01^{\mathrm{b}}$ \\
\hline T5 & $9,73 \pm 0,01^{\mathrm{d}}$ & $4,42 \pm 0,01^{\mathrm{e}}$ & $2,76 \pm 0,00^{\mathrm{e}}$ & $10,82 \pm 0,01^{\mathrm{e}}$ & $58,63 \pm 0,00^{\mathrm{e}}$ \\
\hline T6 & $9,82 \pm 0,00^{\mathrm{b}}$ & $4,64 \pm 0,01^{\mathrm{b}}$ & $3,12 \pm 0,03^{\mathrm{c}}$ & $12,18 \pm 0,00^{\mathrm{b}}$ & $55,06 \pm 0,00^{\mathrm{h}}$ \\
\hline T7 & $9,64 \pm 0,01^{\mathrm{f}}$ & $4,32 \pm 0,00^{\mathrm{f}}$ & $2,34 \pm 0,00^{\mathrm{g}}$ & $9,59 \pm 0,00^{\mathrm{g}}$ & $62,27 \pm 0,02^{\mathrm{c}}$ \\
\hline T8 & $9,75 \pm 0,00^{\mathrm{d}}$ & $4,20 \pm 0,00^{\mathrm{h}}$ & $2,98 \pm 0,00^{\mathrm{d}}$ & $10,86 \pm 0,01^{\mathrm{d}}$ & $57,76 \pm 0,00^{\mathrm{f}}$ \\
\hline T9 & $9,67 \pm 0,01^{\mathrm{e}}$ & $4,49 \pm 0,00^{\mathrm{d}}$ & $2,40 \pm 0,02^{\mathrm{f}}$ & $10,09 \pm 0,00^{\mathrm{f}}$ & $61,23 \pm 0,00^{\mathrm{d}}$ \\
\hline T
\end{tabular}

T: Tratamiento; HA: Harina de Arroz; HM: Harina de Maíz; PZD: Pulpa de zapallo deshidratada. Los valores corresponden al promedio \pm DE: Desviación Estândar $(\mathrm{n}=3)$. Letras diferentes en la misma columna indican diferencias significativas entre tratamientos $(p<0,05)$.

\subsection{Esfuerzo a la fractura y calidad de cocción}

La elaboración de productos libres de gluten se dificulta debido a lo complejo que es suplir la viscoelasticidad que ofrece el gluten en las masas. En este trabajo, los principales componentes que proporcionan viscoelasticidad a la masa fueron HA y HM, las cuales son fuente principal de almidón y se empleó PZD como agente enriquecedor de proteína, minerales y fibra dietaria. Adicionalmente, se usó el huevo con el fin de reforzar el contenido proteico y de esta forma reemplazar parcialmente la red viscoelástica de la que carece la formulación. Lo anterior se ve reflejado en los resultados obtenidos para esfuerzo de fractura y calidad de cocción (Tabla 3).

El tratamiento tuvo un efecto significativo $(p<0,05)$ en el esfuerzo a la fractura, siendo mayor en los tratamientos $2(\mathrm{PZD}=0,10), 4(\mathrm{PZD}=0,10)$ y $7(\mathrm{PZD}=0,1375)$, y menor en los tratamientos 9 $(\mathrm{PZD}=0,1375), 1(\mathrm{PZD}=0,25)$ y $5(\mathrm{PZD}=0,1750)($ ver Tabla 3$)$. Por lo tanto, se evidencia una tendencia de aumento del esfuerzo a la fractura con la disminución PZD en la formulación. Lo anterior, se corroboró con la correlación de Pearson, obteniéndose un valor de $-0,593$ con una significancia muy baja $(0,092)$ dada la variabilidad obtenida entre tratamientos. A diferencia de lo obtenido en este estudio, Mirhosseini et al. (2015) reportaron un aumento de la dureza con la adición de pulpa de zapallo deshidratada a la formulación, informando que este tipo de harina al ser incorporada en productos de panadería muestra una resistencia relativamente alta a la rotura. Dicha diferencia puede deberse al mayor contenido de grasa $(2,18 \mathrm{~g} / 100 \mathrm{~g})$ y humedad $(11,47 \mathrm{~g} / 100 \mathrm{~g})$ que presentó PZD de este estudio respecto a su contenido de grasa $(1,17 \mathrm{~g} / 100 \mathrm{~g})$ y humedad $(10,54 \mathrm{~g} / 100 \mathrm{~g})$. Por un lado, la grasa al tener un efecto plastificante, suavizando la masa y lubricándola, conduce a que el producto sea fácilmente fracturable. Por otro lado, el agua determina la naturaleza de las interacciones entre los distintos constituyentes de la receta, lo que influye en la fuerza de la masa, siendo menor a medida que el contenido de humedad aumenta (Coultate, 1984).

Tabla 3. Esfuerzo a la fractura y calidad de cocción de los tallarines libres de gluten enriquecidos con pulpa de zapallo deshidratada.

\begin{tabular}{|c|c|c|c|c|}
\hline $\mathbf{T}$ & Esfuerzo a la fractura $\left(\mathrm{N} / \mathrm{mm}^{2}\right)^{1}$ & Tiempo de cocción $(\mathrm{min})^{2}$ & Ganancia de peso $(\mathrm{g} / \mathrm{g})^{2}$ & Sólidos perdidos $(\mathrm{g} / \mathrm{g})^{2}$ \\
\hline 1 & $1,41 \pm 0,34^{\mathrm{h}}$ & $9,70 \pm 0,00^{\mathrm{d}}$ & $1,38 \pm 0,10^{\mathrm{ab}}$ & $0,06 \pm 0,00^{\mathrm{d}}$ \\
\hline 2 & $1,92 \pm 0,42^{\mathrm{b}}$ & $12,97 \pm 0,00^{\mathrm{a}}$ & $1,61 \pm 0,06^{\mathrm{ab}}$ & $0,07 \pm 0,00^{\mathrm{c}}$ \\
\hline 3 & $1,59 \pm 0,35^{\mathrm{f}}$ & $7,25 \pm 0,35^{\mathrm{f}}$ & $1,32 \pm 0,12^{\mathrm{b}}$ & $0,07 \pm 0,00^{\mathrm{c}}$ \\
\hline 4 & $2,16 \pm 0,21^{\mathrm{a}}$ & $10,35 \pm 0,00^{\mathrm{c}}$ & $1,38 \pm 0,25^{\mathrm{ab}}$ & $0,05 \pm 0,01^{\mathrm{e}}$ \\
\hline 5 & $1,53 \pm 0,35^{\mathrm{g}}$ & $6,90 \pm 0,00^{\mathrm{g}}$ & $1,47 \pm 0,07^{\mathrm{ab}}$ & $0,04 \pm 0,00^{\mathrm{f}}$ \\
\hline 6 & $1,68 \pm 0,56^{\mathrm{d}}$ & $5,12 \pm 0,00^{\mathrm{h}}$ & $1,23 \pm 0,12^{\mathrm{b}}$ & $0,08 \pm 0,00^{\mathrm{b}}$ \\
\hline 7 & $1,64 \pm 0,77^{\mathrm{e}}$ & $10,87 \pm 0,00^{\mathrm{b}}$ & $1,78 \pm 0,20^{\mathrm{a}}$ & $0,09 \pm 0,00^{\mathrm{a}}$ \\
\hline 8 & $1,70 \pm 0,68^{\mathrm{c}}$ & $8,23 \pm 0,00^{\mathrm{e}}$ & $1,29 \pm 0,14^{\mathrm{b}}$ & $0,09 \pm 0,00^{\mathrm{a}}$ \\
\hline 9 & $1,40 \pm 0,48^{\mathrm{i}}$ & $9,57 \pm 0,00^{\mathrm{d}}$ & $1,44 \pm 0,08^{\mathrm{ab}}$ & $0,06 \pm 0,00^{\mathrm{d}}$ \\
\hline
\end{tabular}

$\mathrm{T}$ : Tratamiento. ${ }^{1}$ Los datos corresponden al promedio $\pm \mathrm{DE}(\mathrm{n}=5) .{ }^{2}$ Los datos corresponden al promedio \pm DE: Desviación Estándar $(\mathrm{n}=3)$. Letras diferentes en la misma columna indican diferencias significativas entre tratamientos $(p<0,05)$ 
En cuanto a la calidad de cocción, se detectaron diferencias significativas $(p<0,05)$ en el tiempo de cocción, ganancia de peso y sólidos perdidos entre tratamientos (ver Tabla 4). El tiempo de cocción fue significativamente mayor para los tratamientos $2(\mathrm{PZD}=0,10), 7(\mathrm{PZD}=0,1375)$ y $4(\mathrm{PZD}=0,10)$ con valores de 12,$97 ; 10,87$ y 10,35 min respectivamente, y significativamente menor para los tratamientos 6 $(\mathrm{PZD}=0,2125 \mathrm{~g} / \mathrm{g}), 5(\mathrm{PZD}=0,1750 \mathrm{~g} / \mathrm{g})$ y $3(\mathrm{PZD}=0,25 \mathrm{~g} / \mathrm{g})$ con valores de 5,12; 6,90 y 7,25 min respectivamente. Es decir, a medida que aumentó PZD en la formulación, disminuyó el tiempo de cocción. Adicionalmente, se evidenció que los tratamientos con mayor tiempo de cocción presentaron mayor ganancia de peso; registrándose mayores valores en los tratamientos 7, 2 y 5 que corresponden a formulaciones con PZD $>0,10$.

Respecto a los sólidos perdidos en el agua de cocción, fue mayor en los tratamientos 7 y $8(0,09 \mathrm{~g} / \mathrm{g})$ y significativamente menor en el tratamiento $5(\mathrm{SP}=0,04 \mathrm{~g} / \mathrm{g})(p<0,05)$. De forma general, se evidenció un aumento de los sólidos perdidos en el agua de cocción por el aumento de PZD, sin embargo, esta tendencia no fue clara, debido al efecto compensatorio que proporcionaron las demás harinas (HA y HM) a la formulación.

López-Mejía et al. (2019) reportaron un comportamiento similar para pastas enriquecidas con pulpa de zapallo deshidratada, disminuyendo alrededor de 4 min el tiempo de cocción al aumentar la proporción de PZD desde $0 \mathrm{~g} / 100 \mathrm{~g}$ hasta $10 \mathrm{~g} / 100 \mathrm{~g}$ de harina. En este estudio la disminución del tiempo alcanzó los 8 min al aumentar PZD desde $10 \mathrm{~g} / 100 \mathrm{~g}$ hasta $25 \mathrm{~g} / 100 \mathrm{~g}$ de harina. Así mismo, las formulaciones con mayor tiempo de cocción (formulaciones con menor proporción de PZD), registraron mayor ganancia de peso. La disminución del tiempo de cocción debido a la incorporación de materias primas ricas en fibra ya ha sido explicada por Manthey \& Schorno (2002), atribuyendo este comportamiento a la interrupción de la matriz formada por el gluten y el almidón, por la fibra; esto conlleva a que el agua se absorba fácilmente.

Por otro lado, Minarovičová et al. (2017) similar a lo analizado en este estudio, reportaron una disminución en el tiempo de cocción de 7 a 5,9 min cuando el nivel de adición de PZD aumenta de 0 a $10 \%$. Adicionalmente, se concluye que la adición de pulpa de zapallo aumenta los sólidos perdidos en el agua de cocción, debido a la ruptura de la matriz proteína-almidón y a la distribución heterogénea del agua dentro de la pasta por la tendencia competitiva de la fibra, evitando la hinchazón del almidón. Esto se debe a la falta de una red de gluten en este tipo de pastas y al aumento del contenido de fibra en la formulación, aportada por PZD (Marti \& Pagani, 2013).

\subsection{Microestructura}

En la Figura 1 se muestran las microestructuras de los tallarines libres de gluten enriquecidos con PZD a diferentes tratamientos, con aumentos de $30 \mathrm{x}, 200$ x y $800 \mathrm{x}$. Las Figuras $1 \mathrm{a}$ y $1 \mathrm{~b}$ corresponden a los tratamientos 2 y 4, cuyas formulaciones contienen la menor proporción de PZD (10 g/100 g de harina). Las Figuras 1c y $2 \mathrm{~d}$ corresponden a los tratamientos 1 y 3 , cuyas formulaciones contienen la mayor proporción de PZD ( $25 \mathrm{~g} / 100 \mathrm{~g}$ de harina). En ellas se evidencia el aumento la porosidad de la sección transversal debido al aumento de la proporción de PZD en la formulación. Así mismo, también aumentó el tamaño de los poros, siendo para el tratamiento 2 de 6,14 $\pm 0,98 \mu \mathrm{m}$, para el tratamiento 4 de 5,73 $\pm 0,63 \mu \mathrm{m}$; para el tratamiento 1 de $8,72 \pm 0,70 \mu \mathrm{m}$ y para el tratamiento 3 de $9,92 \pm 0,95 \mu \mathrm{m}$. De acuerdo con los resultados obtenidos para la correlación de Pearson $(p<0,05)$, el tamaño del poro tiene una relación negativa con el esfuerzo a la fractura, con un valor de $-0,894(p=0,000)$. Por otro lado, se correlacionó el tamaño del poro con la proporción de PZD en la muestra obteniéndose una relación positiva con un valor de $0,902(p=0,000)$. Adicionalmente, se observó una correlación negativa entre el nivel de HM en la formulación de TLG, con el tamaño del poro (- 0,552$)$; sin embargo, esta no fue significativa $(p=0,063)$. Um estudio similar en el que se evidencia la afectación en la microestructura de la red de pastas alimenticias por la incorporación de harinas no convencionales ha sido reportado en la literatura. Fiorda et al. (2013), 
Optimización de la formulación de tallarines libres de gluten enriquecidos con pulpa de zapallo deshidratada empleando el método de diseño de mezclas

López-Mejía, N., \& Morales Posada, N. B.

evidenciaron que a medida que aumentaban la adición de harina de yuca a la formulación, se presentaban grietas, generando falta de continuidad en la estructura y una mayor porosidad, lo que atribuyeron a la menor proporción de proteína y menor cantidad de harina pregelatinizada. Para este estudio el aumento de la porosidad no se debería a una menor proporción de proteína en la formulación de TLG, sino a la disminución del contenido de almidón y al aumento del contenido de fibra dietaria.

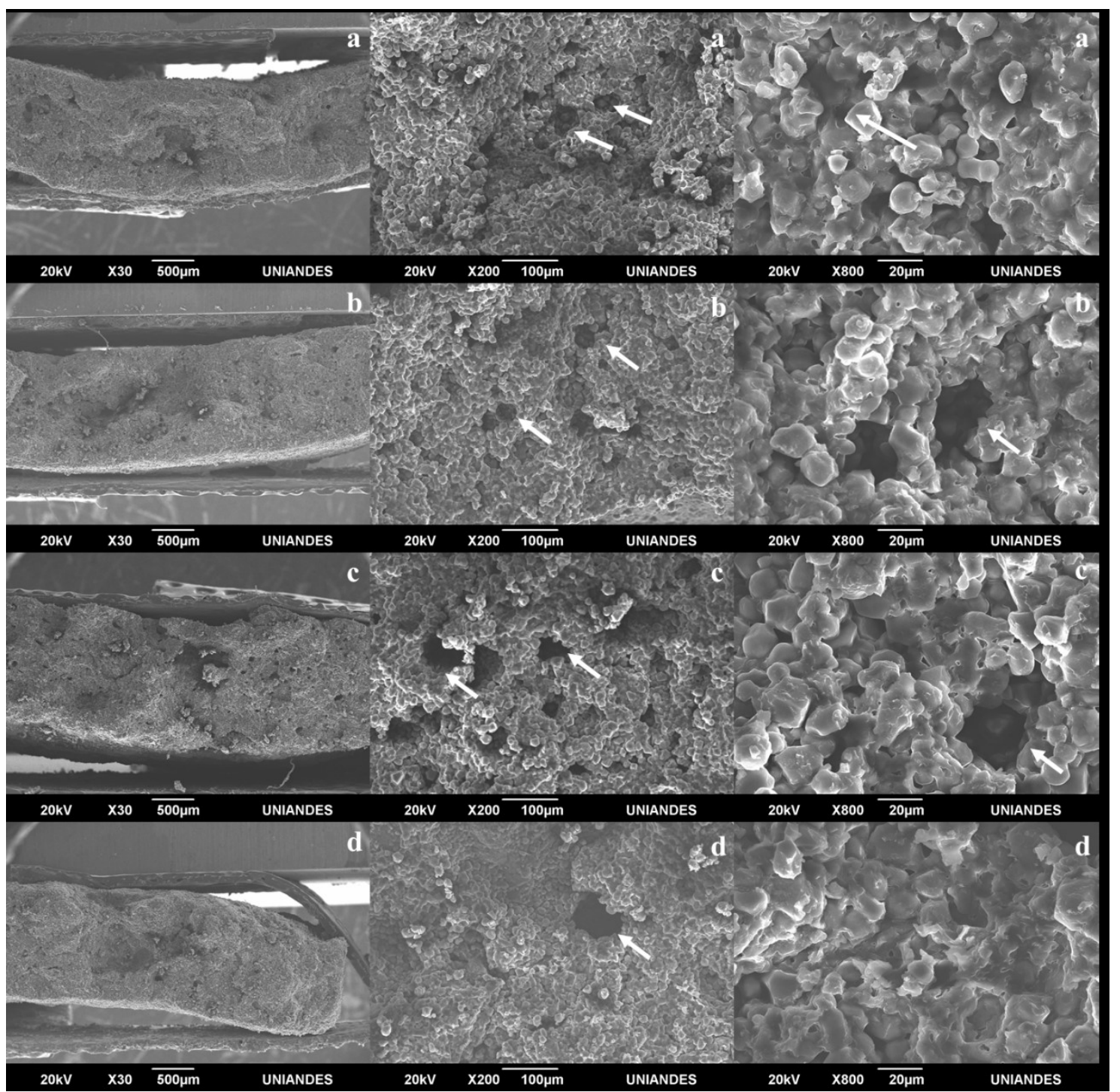

Figura 1. Micrografías que se refieren a la fractura de los tallarines de los tratamientos 2 (a), 4 (b), 1 (c) y 3 (d) con aumentos de 30 x 200 x y 800 x. Las flechas indican los poros de las sección transversal de los tallarines fracturados.

\subsection{Color}

El color es uno de los parámetros fundamentales que determinan la elección de los consumidores sobre un producto. Este puede verse afectado por las condiciones de procesamiento, formulaciones y/o a la presencia de ingredientes específicos, incluyendo los agregados para mejorar el valor nutricional (Marti et al., 2016; Petitot et al., 2010). La adición de las diferentes harinas usadas en este estudio resultó tener un efecto significativo en el color $(p<0,05)$ (Tabla 4), en particular debido al alto contenido de carotenoides (no cuantificados en este estudio) de PZD, lo que le proporciona a la mezcla una tonalidad naranja. 
$\mathrm{Al}$ observar las coordenadas $\mathrm{CIE}_{\mathrm{L}^{*} \mathrm{a}^{*} \mathrm{~b}^{*}}$ obtenidas para los diferentes tratamientos (Tabla 4), se evidencia una disminución significativa en el valor de las coordenadas $L^{*}(67,85$ a 64,02$)$ y b* $(57,42$ a 50,60$)$ y un aumento del valor de $a^{*}(14,49$ a 17,81) con el incremento de PZD en la formulación. Semejante a lo obtenido en este estudio, Mirhosseini et al. (2015) reportan que el valor de a* fue mayor $(1,35$ a 2,07$)$ y el valor de $b^{*}$ menor $(52,01$ a 51,96) en pastas alimenticias con PZD alto $(25$ y 50\%), debido al aumento del color naranja en la mezcla. Respecto a resultados obtenidos para pastas enriquecidas con otro tipo de harina vegetal; Marengo et al. (2018), desarrollaron PLG enriquecidas con papa de pulpa naranja; evidenciando que al aumentar la proporción de PZD en la formulación, disminuye la luminosidad ( $\left.\mathrm{L}^{*}\right)$ y aumenta el valor de $\mathrm{a}^{*} \mathrm{y} \mathrm{b}^{*}$, así como lo observado por Morreale et al. (2019), para PLG con harina de arroz y maíz $\left(L^{*}=73,19 \pm 0.21 ; a^{*}=3,97 \pm 0.21 \mathrm{yb}^{*}=29,70 \pm 0,59\right)$.

Tabla 4. Análisis colorimétrico de los tallarines libres de gluten enriquecidos con pulpa de zapallo deshidratada.

\begin{tabular}{cccc}
\hline $\mathbf{T}$ & $\mathbf{L}^{*}$ & $\mathbf{a}^{*}$ & $\mathbf{b}^{*}$ \\
\hline $\mathbf{1}$ & $65,60 \pm 0,27^{\mathrm{d}}$ & $17,71 \pm 0,62^{\mathrm{c}}$ & $55,81 \pm 2,99^{\mathrm{c}}$ \\
\hline $\mathbf{2}$ & $65,48 \pm 1,08^{\mathrm{e}}$ & $17,63 \pm 0,82^{\mathrm{d}}$ & $54,92 \pm 3,24^{\mathrm{d}}$ \\
\hline $\mathbf{3}$ & $64,02 \pm 0,20^{\mathrm{g}}$ & $17,29 \pm 0,51 \mathrm{e}$ & $49,14 \pm 1,87 \mathrm{~g}$ \\
\hline $\mathbf{5}$ & $67,85 \pm 0,36^{\mathrm{a}}$ & $14,70 \pm 0,64^{\mathrm{h}}$ & $56,40 \pm 1,94^{\mathrm{b}}$ \\
\hline $\mathbf{6}$ & $65,35 \pm 0,56^{\mathrm{f}}$ & $17,81 \pm 0,72^{\mathrm{b}}$ & $57,42 \pm 1,90^{\mathrm{a}}$ \\
\hline $\mathbf{7}$ & $65,75 \pm 0,95^{\mathrm{c}}$ & $15,43 \pm 0,43^{\mathrm{g}}$ & $48,79 \pm 0,59^{\mathrm{h}}$ \\
\hline $\mathbf{8}$ & $67,61 \pm 0,28^{\mathrm{b}}$ & $14,49 \pm 0,41^{\mathrm{i}}$ & $50,60 \pm 2,12^{\mathrm{f}}$ \\
\hline $\mathbf{9}$ & $62,17 \pm 1,00^{\mathrm{i}}$ & $17,94 \pm 1,12^{\mathrm{a}}$ & $50,71 \pm 2,10^{\mathrm{e}}$ \\
\hline
\end{tabular}

T: Tratamiento. Los datos corresponden al promedio \pm DE: Desviación Estándar $(n=5)$. Letras diferentes en la misma columna indican diferencias significativas entre tratamientos $(p<0,05)$.

\subsection{Análisis sensorial}

En la Figura 2 se muestra el promedio del puntaje obtenido para cada parámetro medido en la evaluación sensorial de aceptación, de TLG desarrollados con PZD, allí se observa que los parámetros con mayor puntaje son el color y el olor, los parámetros de textura son los menos aceptados (puntaje $<2$ ). Por otro lado, se evidencia una disminución en la aceptación de los tallarines a medida que aumenta la proporción de PZD en la formulación. Sin embargo, según el análisis de varianza ANOVA y la prueba Tukey realizada, no hay diferencias significativas entre tratamientos $(p>0,05)$.

De manera similar, Minarovičová et al. (2017) reportaron que al aumentar el nivel de adición de PZD a la formulación de pastas alimenticias, decrece el puntaje de sabor y textura y aumenta el puntaje de color y olor, también señalaron que la formulación con mayor aceptación es la desarrollada con un nivel de adición de $10 \%$ PZD, dicha diminución en la aceptación sensorial de productos sin gluten se debe al aumento del contenido fibra dietaria, y al sabor vegetal residual que proporciona PZD (Kohajdová et al., 2011). 


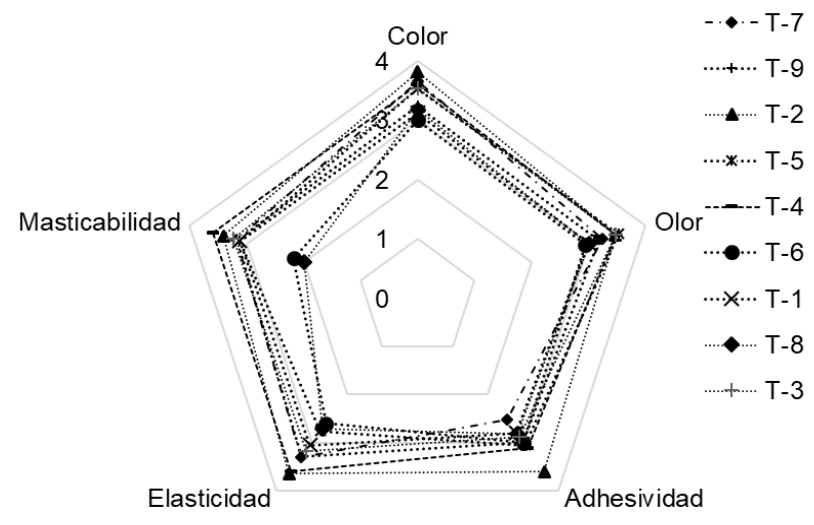

Figura 2. Aceptación sensorial de los tallarines libres de gluten enriquecidos con pulpa de zapallo deshidratada.

Los valores corresponden al promedio de $n=30$. (T: Tratamiento).

\subsection{Optimización de las formulaciones}

De acuerdo con los resultados de análisis de varianza para cada variable de respuesta con cada uno de los términos evaluados (lineal, cuadrático, cúbico especial y cúbico completo), las variables mejor explicadas por los modelos $\left(\mathrm{R}^{2}>0,8\right.$ y $\left.p<0,05\right)$ fueron: adhesividad sensorial, masticabilidad sensorial y ganancia de peso. Para las demás variables no se obtuvieron modelos con alta significancia estadística (Tabla 5). El término con mayor grado ajuste a la variable adhesividad sensorial, fue el cúbico especial. Para la masticabilidad sensorial y para la ganancia de peso, fue el cuadrático. Dichas variables fueron usadas para hallar la formulación óptima.

En la Figura 3, se muestra el comportamiento de cada una de las variables, resaltándose el punto máximo para cada una de ellas (adhesividad: 5,03; masticabilidad sensorial 3,51 y ganancia: 1,50 g/g respectivamente). Teniendo en cuenta que a cada una se le asignó un peso de 1 y una importancia de 3,2 y 1 , respectivamente; se obtuvo como formulación óptima aquella con proporciones de $\mathrm{PZD}=0,10$; $\mathrm{HÁ}=0,3859$ y $\mathrm{HM}=0,5141 \mathrm{~g} / \mathrm{g}$ harina. Las gráficas de contorno de los atributos individuales fueron superpuestas para obtener una región óptima (Figura 3b) donde se cumplieron las condiciones especificadas en los parámetros adhesividad sensorial, masticabilidad sensorial y ganancia de peso. De las formulaciones desarrolladas la que más se asemejó a dicho resultado fue la correspondiente al tratamiento 2 .

Tabla 5. Análisis de varianza de los términos lineal, cuadrático y cubico especial para cada parámetro estudiado.

\begin{tabular}{|c|c|c|c|c|c|}
\hline Parámetros & Término & $\mathbf{F}$ & $\mathbf{P}$ & $\mathbf{R}^{2}$ & $\mathbf{R}^{2}$ ajustado \\
\hline \multirow{4}{*}{ Color $^{1}$} & Lineal & 1,88 & 0,232 & 0,3858 & 0,1810 \\
\hline & Cuadrático & 0,59 & 0,664 & 0,6131 & 0,0000 \\
\hline & Cúbico especial & 0,77 & 0,474 & 0,7202 & 0,0000 \\
\hline & Cúbico completo & 0,71 & 0,555 & 0,8361 & 0,0000 \\
\hline \multirow{4}{*}{ Olor $^{1}$} & Lineal & 5,65 & 0,042 & 0,6531 & 0,5375 \\
\hline & Cuadrático & 0,73 & 0,598 & 0,7998 & 0,4662 \\
\hline & Cúbico especial & 3,71 & 0,194 & 0,9299 & 0,7196 \\
\hline & Cúbico completo & 0,03 & 0,894 & 0,9318 & 0,4545 \\
\hline \multirow{4}{*}{ Adhesividad $^{1}$} & Lineal & 2,22 & 0,190 & 0,4255 & 0,2340 \\
\hline & Cuadrático & 0,32 & 0,812 & 0,5649 & 0,0000 \\
\hline & Cúbico especial & $\mathbf{5 7 , 8 7}$ & $\mathbf{0 , 0 1 7}$ & 0,9855 & 0,9419 \\
\hline & Cúbico completo & 1,34 & 0,454 & 0,9938 & 0,9503 \\
\hline \multirow{4}{*}{ Elasticidad $^{1}$} & Lineal & 0,94 & 0,441 & 0,2389 & 0,0000 \\
\hline & Cuadrático & 0,82 & 0,563 & 0,5820 & 0,0000 \\
\hline & Cúbico especial & 0,59 & 0,521 & 0,6778 & 0,0000 \\
\hline & Cúbico completo & 1,08 & 0,487 & 0,8452 & 0,0000 \\
\hline
\end{tabular}


Optimización de la formulación de tallarines libres de gluten enriquecidos con pulpa de zapallo deshidratada empleando el método de diseño de mezclas

López-Mejía, N., \& Morales Posada, N. B.

Tabla 5. Continuación..

\begin{tabular}{|c|c|c|c|c|c|}
\hline Parámetros & Término & $\mathbf{F}$ & $\mathbf{P}$ & $\mathbf{R}^{2}$ & $\mathbf{R}^{2}$ ajustado \\
\hline \multirow{4}{*}{ Masticabilidad $^{1}$} & Lineal & 2,84 & 0,136 & 0,4862 & 0,3150 \\
\hline & Cuadrático & 100,85 & 0,002 & 0,9950 & 0,9865 \\
\hline & Cúbico especial & 7,94 & 0,106 & 0,9990 & 0,9959 \\
\hline & Cúbico completo & 1,41 & 0,445 & 0,9996 & 0,9966 \\
\hline \multirow{4}{*}{$\begin{array}{l}\text { Tiempo de } \\
\text { cocción }\end{array}$} & Lineal & 2,34 & 0,177 & 0,4381 & 0,2508 \\
\hline & Cuadrático & 4,21 & 0,134 & 0,8922 & 0,7125 \\
\hline & Cúbico especial & 0,33 & 0,623 & 0,9075 & 0,6300 \\
\hline & Cúbico completo & 13,41 & 0,170 & 0,9936 & 0,9486 \\
\hline \multirow{4}{*}{$\begin{array}{c}\text { Ganancia de } \\
\text { peso }\end{array}$} & Lineal & 0,79 & 0,496 & 0,2083 & 0,0000 \\
\hline & Cuadrático & 11,03 & 0,040 & 0,9342 & 0,8245 \\
\hline & Cúbico especial & 0,02 & 0,898 & 0,9349 & 0,7394 \\
\hline & Cúbico completo & 0,01 & 0,941 & 0,9354 & 0,4833 \\
\hline \multirow{4}{*}{ Sólidos perdidos } & Lineal & 0,56 & 0,599 & 0,1571 & 0,0000 \\
\hline & Cuadrático & 0,21 & 0,884 & 0,3032 & 0,0000 \\
\hline & Cúbico especial & 13,30 & 0,068 & 0,9089 & 0,6358 \\
\hline & Cúbico completo & 0,09 & 0,812 & 0,9166 & 0,3332 \\
\hline \multirow{4}{*}{ Proteína } & Lineal & 0,80 & 0,493 & 0,2102 & 0,0000 \\
\hline & Cuadrático & 1,16 & 0,454 & 0,6337 & 0,0232 \\
\hline & Cúbico especial & 0,01 & 0,920 & 0,6360 & 0,0000 \\
\hline & Cúbico completo & 4,76 & 0,274 & 0,9368 & 0,4945 \\
\hline \multirow{4}{*}{ Almidón } & Lineal & 0,73 & 0,518 & 0,1967 & 0,0000 \\
\hline & Cuadrático & 1,13 & 0,462 & 0,6221 & 0,0000 \\
\hline & Cúbico especial & 0,01 & 0,930 & 0,6239 & 0,0000 \\
\hline & Cúbico completo & 5,49 & 0,257 & 0,9421 & 0,5366 \\
\hline \multirow{4}{*}{ Cenizas } & Lineal & 0,48 & 0,641 & 0,1378 & 0,0000 \\
\hline & Cuadrático & 0,87 & 0,544 & 0,5393 & 0,0000 \\
\hline & Cúbico especial & 0,01 & 0,937 & 0,5411 & 0,0000 \\
\hline & Cúbico completo & 13,61 & 0,169 & 0,9686 & 0,7487 \\
\hline \multirow{4}{*}{ Fibra dietaria } & Lineal & 0,96 & 0,435 & 0,2424 & 0,0000 \\
\hline & Cuadrático & 1,37 & 0,401 & 0,6803 & 0,1475 \\
\hline & Cúbico especial & 0,08 & 0,806 & 0,6924 & 0,0000 \\
\hline & Cúbico completo & 2,82 & 0,342 & 0,9195 & 0,3559 \\
\hline \multirow{4}{*}{$\begin{array}{l}\text { Esfuerzo a la } \\
\text { fractura* }\end{array}$} & Lineal & 1,73 & 0,254 & 0,3664 & 0,1552 \\
\hline & Cuadrático & 5,57 & 0,096 & 0,9036 & 0,7430 \\
\hline & Cúbico especial & 0,01 & 0,923 & 0,9042 & 0,6168 \\
\hline & Cúbico completo & 0,16 & 0,76 & 0,9172 & 0,3378 \\
\hline \multirow{4}{*}{$\mathbf{L}^{*}$} & Lineal & 1,04 & 0,409 & 0,2579 & 0,0106 \\
\hline & Cuadrático & 0,10 & 0,956 & 0,3240 & 0,0000 \\
\hline & Cúbico especial & 0,11 & 0,771 & 0,3594 & 0,0000 \\
\hline & Cúbico completo & 0,00 & 0,983 & 0,3599 & 0,0000 \\
\hline \multirow{4}{*}{$a^{*}$} & Lineal & 0,91 & 0,451 & 0,2330 & 0,0000 \\
\hline & Cuadrático & 0,29 & 0,833 & 0,4042 & 0,0000 \\
\hline & Cúbico especial & 0,70 & 0,49 & 0,5589 & 0,0000 \\
\hline & Cúbico completo & 0,02 & 0,908 & 0,5682 & 0,0000 \\
\hline \multirow{4}{*}{$\mathbf{b}^{*}$} & Lineal & 0,45 & 0,659 & 0,1298 & 0,0000 \\
\hline & Cuadrático & 0,26 & 0,849 & 0,3116 & 0,0000 \\
\hline & Cúbico especial & 7,35 & 0,113 & 0,8527 & 0,4110 \\
\hline & Cúbico completo & 4,16 & 0,29 & 0,9715 & 0,7716 \\
\hline
\end{tabular}

${ }^{1}$ Atributos sensoriales medidos a los tallarines cocidos. F: Estadístico de prueba; $\mathrm{P}$ : significancia del modelo; $\mathrm{R}^{2}$ y $\mathrm{R}^{2}$ ajustado: Coeficientes de determinación $(p<0,05)$. En negrita se resaltan las variables mejor explicadas por los modelos $(\mathrm{R} 2>0,8$ y $\mathrm{p}<0,05)$. 
En la Tabla 6 se muestran los coeficientes de cada modelo obtenido, además de la significancia de las variables independientes (PZD, HA y HM) y sus interacciones. En ella se evidencia que las variables de forma independiente no tienen un efecto significativo sobre las variables de respuesta; sin embargo, las interacciones entre ellas sí. Resultados similares fueron obtenidos por Codina \& Mironeasa (2016) para panes libres de gluten enriquecidos con pulpa de zapallo deshidratada. Estos evidenciaron que las harinas evaluadas (pulpa de zapallo, semilla de zapallo y harina de trigo), de forma independiente no influyen significativamente en la porosidad del pan, mientras que sus interacciones sí.
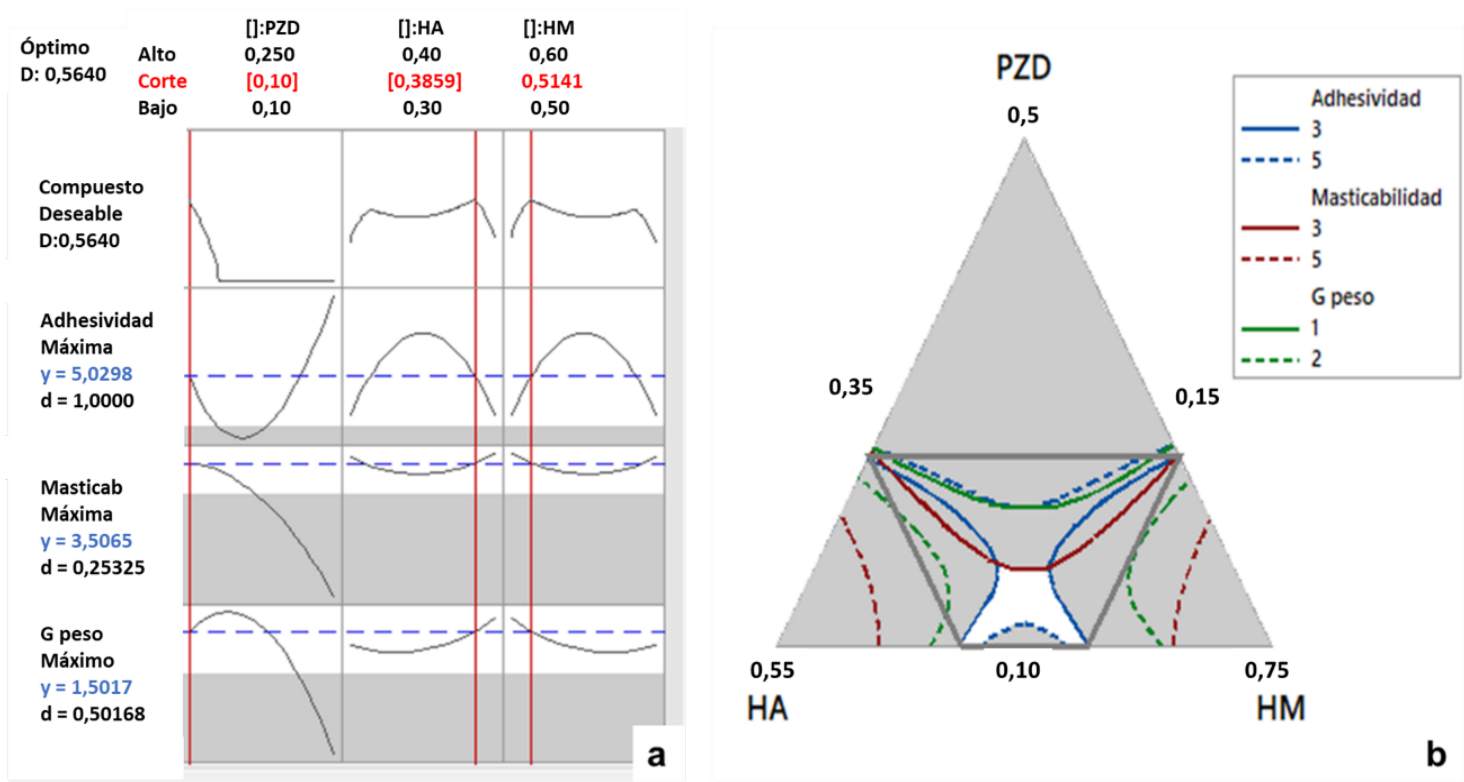

Figura 3. Optimización de la respuesta. a) Gráficas de optimización de la respuesta por cada variable y b) Parcelas de contorno superpuestas que muestran la región óptima (área sin sombrear). PZD: Pulpa de zapallo deshidratada; HA: Harina de Arroz; HM: Harina de Maíz; G peso: Ganancia de Peso (g/g).

Tabla 6. Coeficientes de regresión de los modelos predichos para parámetros adhesividad, masticabilidad sensorial y ganancia de peso.

\begin{tabular}{cccc}
\hline Componente & Adhesividad sensorial & $\begin{array}{c}\text { Parámetro } \\
\text { Masticabilidad sensorial }\end{array}$ & Ganancia de peso (g/g) \\
\hline PZD & -357 & $-68,9$ & $-123,9$ \\
\hline HA & -666 & 45,3 & 37,5 \\
\hline HM & -278 & 21,1 & 10,3 \\
\hline PZD*HA & $2798^{*}$ & 60,9 & $131,0^{*}$ \\
\hline PZD*HM & $1499^{*}$ & 61,1 & $162,8^{*}$ \\
\hline PZD*HA*HM & $-6430^{*}$ & - & $-97,5^{*}$ \\
\hline HA*HM & - & $-118,1^{*}$ & $0,040^{*}$ \\
\hline P-valor & $0,017^{*}$ & $0,002^{*}$ & 0,9342 \\
\hline $\mathrm{R}^{2}$ & 0,9855 & 0,9950 & 0,8245 \\
\hline $\mathrm{R}^{2}$ ajustado & 0,9419 & 0,9865 & $\mathrm{~s}$ \\
\hline
\end{tabular}

PZD: Pulpa de Zapallo Deshidratada; HA: Harina de Arroz y HM: Harina de Maíz. *Significante en el nivel de $\mathrm{p}<0,05$. P: significancia del modelo; $\mathrm{R}^{2}$ y $\mathrm{R}^{2}$ ajustado: Coeficientes de determinación $(\mathrm{p}<0,05)$.

\section{Conclusiones}

Con este estudio se lograron evidenciar diferencias significativas entre tratamientos, en cuanto a los parámetros que determinan la calidad de los tallarines libres de gluten; sin embargo, no se hallaron diferencias en cuanto la aceptación sensorial. 
Se lograron obtener modelos matemáticos que describen variables de respuesta como adhesividad sensorial, masticabilidad sensorial y ganancia de peso, con una significancia $p<0,05$ y valores de $\mathrm{R}^{2}$ y $\mathbf{R}^{2}$ ajustado altos $(>0,8)$. Para la adhesividad sensorial el término que mejor se ajustó fue el cúbico completo y para las otras dos variables fue el cuadrático. Finalmente, la formulación seleccionada como la óptima fue la correspondiente al tratamiento 2 (valores predichos: $\mathrm{PZD}=0,10 ; \mathrm{HA}=0,3859$ y $\mathrm{HM}=0,5141 \mathrm{~g} / \mathrm{g}$ harina; valores experimentales $(\mathrm{PZD}=0,10 ; \mathrm{HA}=0,40$ y $\mathrm{HM}=0,50)$ ).

\section{Referencias}

American Association of Cereal Chemists - AACC. (2000). AACC method 66-50: Pasta and noodle cooking quality: Firmness. St. Paul: AACC.

American Association of Cereal Chemists - AACC. (2001). AACC method 08-01: Ash basic method. St. Paul: AACC.

ASTM International. (2016). ASTM D2244 -16: Standard practice for calculation of color tolerances and color differences from instrumentally measured color coordinates. West Conshohocken: ASTM International.

Association of Official Analytical Chemists - AOAC International. (2003). AOAC 985-29: Enzimic gravimetric method for determination of dietary fiber in oat and corn grains (Vol. 53). Gaithersburg: AOAC

Bastos, G. M., Soares Júnior, M. S., Caliari, M., Araujo Pereira, A. L., De Morais, C. C., \& Campos, M. R. H. (2016). Physical and sensory quality of gluten-free spaghetti processed from amaranth flour and potato pulp. Lebensmittel-Wissenschaft + Technologie, 65, 128-136. http://dx.doi.org/10.1016/j.Iwt.2015.07.067

Camelo-Méndez, G., Tovar, J., \& Bello-Pérez, L. A. (2018). Influence of blue maize flour on gluten-free pasta quality and antioxidant retention characteristics. Journal of Food Science and Technology, 55(7), 2739-2748. PMid:30042590. http://dx.doi.org/10.1007/s13197-018-3196-9

Carpenter, R., Lyon, D., \& Hasdell, T. (2009). Análisis sensorial en el desarrollo y control de la calidad de alimentos. Zaragoza: Editorial Acribia.

Catassi, C., Bai, J. C., Bonaz, B., Bouma, G., Calabrò, A., Carroccio, A., Castillejo, G., Ciacci, C., Cristofori, F., Dolinsek, J., Francavilla, R., Elli, L., Green, P., Holtmeier, W., Koehler, P., Koletzko, S., Meinhold, C., Sanders, D., Schumann, M., Schuppan, D., Ullrich, R., Vécsei, A., Volta, U., Zevallos, V., Sapone, A., \& Fasano, A. (2013). Non-celiac gluten sensitivity: The new frontier of gluten related disorders. Nutrients, 5(10), 3839-3853. PMid:24077239. http://dx.doi.org/10.3390/nu5103839

Catassi, C., Gatti, S., \& Lionetti, E. (2015). World perspective and celiac disease epidemiology. Digestive Diseases 33(2), 141146. PMid:25925915. http://dx.doi.org/10.1159/000369518

Codina, G. G., \& Mironeasa, S. (2016). Application of D-optimal mixture design to optimize the wheat T-pumpkin composite flour for bread production. Journal of Faculty of Food Engineering, 15(1), 10-20.

Coultate, T. P. (1984). Food: The chemistry of its components. Cambridge: Royal Society of Chemistry.

Escalada Plaa, M., Ponce, N., Stortz, C., Gerschenson, L., \& Rojas, A. (2007). Composition and functional properties of enriched fiber products obtained from pumpkin (Cucurbita moschata Duchesne ex Poiret). Lebensmittel-Wissenschaft + Technologie, 40(7), 1176-1185. http://dx.doi.org/10.1016/j.Iwt.2006.08.006

Fiorda, F., Soares Junior, M., Silva, F., Grosmann, M. V. E., \& Souto, L. R. F. (2013). Microstructure, texture and color of glutenfree pasta made with amaranth flour, cassava starch and cassava bagasse. Lebensmittel-Wissenschaft + Technologie, 54(1), 132-138. http://dx.doi.org/10.1016/j.Iwt.2013.04.020

Flores-Silva, P., Berrios, J., Pan, J., Osorio-Díaz, P., \& Bello-Pérez, L. (2014). Gluten-free spaghetti made with chickpea, unripe plantain and maize flours: Functional and chemical properties and starch digestibility. International Journal of Food Science \& Technology, 49(9), 1985-1991. http://dx.doi.org/10.1111/ijfs.12529

Instituto Colombiano de Normas Técnicas y Certificación - ICONTEC Internacional. (1973). NTC 668: Alimentos y materias primas: Determinación de contenidos de grasa y fibra cruda. Bogotá: ICONTEC Internacional.

Instituto Colombiano de Normas Técnicas y Certificación - ICONTEC Internacional. (2009). NTC 4566: Productos cárnicos: Determinación del contenido de almidón: Métodos de referencia. Bogotá: ICONTEC Internacional.

International Organization for Standardization - ISO. (2009). ISO 1871: Food and feed products: General guidelines for the determination of nitrogen by the Kjeldahl method (2nd ed.). Geneva: ISO.

Kohajdová, Z., Karovičová, J., Jurasová, M., \& Kukurová, K. (2011). Application of citrus dietary fiber preparations in biscuit production. Journal of Food and Nutrition Research, 50(3), 182-190.

López-Mejía, N., Andrade-Mahecha, M. M., \& Martínez-Correa, H. (2019). Modelamiento matemático de la cinética de secado de espagueti enriquecido con pulpa de zapallo deshidratada (Cucurbita moschata). Revista Udca Actualidad \& Divulgacion Cientifica, 22(1), 1-11. http://dx.doi.org/10.31910/rudca.v22.n1.2019.1151

Manthey, F. A., \& Schorno, A. (2002). Physical and cooking quality of spaghetti made from whole-wheat durum. Cereal Chemistry, 79(4), 504-510. http://dx.doi.org/10.1094/CCHEM.2002.79.4.504 
Optimización de la formulación de tallarines libres de gluten enriquecidos con pulpa de zapallo deshidratada empleando el método de diseño de mezclas

López-Mejía, N., \& Morales Posada, N. B.

Marengo, M., Amoah, I., Carpen, A., Benedetti, S., Zanoletti, M., Buratti, S., Lutterodt, H. E., Johnson, P.-N. T., Manful, J., Marti, A., Bonomi, F., \& lametti, S. (2018). Enriching gluten-free rice pasta with soybean and sweet potato flours. Journal of Food Science and Technology, 55(7), 2641-2648. PMid:30042580. http://dx.doi.org/10.1007/s13197-018-3185-z

Marti, A., D'Egidio, M., \& Pagani, M. (2016). Pasta: Quality testing methods. In C. Wrigley, H. Corke, K. Seetharaman \& F. Faubion. (Eds.), Encyclopedia of cereal grains (2nd ed., pp. 161-165). London: Academic Press. http://dx.doi.org/10.1016/B9780-12-394437-5.00132-7.

Marti, A., Ragg, E., \& Pagani, M. (2018). Effect of processing conditions on water mobility and cooking quality of gluten-free pasta: A Magnetic Resonance Imaging study. Food Chemistry, 266, 17-23. PMid:30381172. http://dx.doi.org/10.1016/j.foodchem.2018.05.057

Marti, A., \& Pagani, M. (2013). What can play the role of gluten in gluten free pasta? Trends in Food Science \& Technology, 31(1), 63-71. http://dx.doi.org/10.1016/j.tifs.2013.03.001

Mastromatteo, M., Chillo, S., lannetti, M., Civica, V., Sepielli, G., \& Del Nobile, M. (2012). Influence of heat treatment on the quality of functional gluten-free spaghetti. Food and Nutrition Sciences, 3(04), 433-440. http://dx.doi.org/10.4236/fns.2012.34062

Mendoza, F., Dejmek, P., \& Aguilera, J. M. (2006). Calibrated color measurements of agricultural foods using image analysis. Postharvest Biology and Technology, 41(3), 285-295. http://dx.doi.org/10.1016/j.postharvbio.2006.04.004

Minarovičová, L., Lauková, M., Kohajdová, Z., Karovičová, J., \& Kuchtová, V. (2017). Effect of pumpkin powder incorporation on cooking and sensory parameters of pasta. Potravinarstvo Slovak Journal of Food Sciences, 11(1), 373-379. http://dx.doi.org/10.5219/743

Mirhosseini, H., Abdul Rashid, N. F., Tabatabaee Amid, B., Cheong, K. W., Kazemi, M., \& Zulkurnain, M. (2015). Effect of partial replacement of corn flour with durian seed flour and pumpkin flour on cooking yield, texture properties, and sensory attributes of gluten free pasta. Lebensmittel-Wissenschaft + Technologie, 63(1), 184-190. http://dx.doi.org/10.1016/j.Iwt.2015.03.078

Morreale, F., Boukid, F., Carini, E., Federici, E., Vittadini, E., \& Pellegrini, N. (2019). An overview of the Italian market for 2015: Cooking quality and nutritional value of gluten-free pasta. International Journal of Food Science \& Technology, 54(3), 780-786. http://dx.doi.org/10.1111/ijfs.13995

Petitot, M., Boyer, L., Minier, C., \& Micard, V. (2010). Fortification of pasta with split pea and faba bean flours: Pasta processing and quality evaluation. Food Research International, 43(2), 634-641. http://dx.doi.org/10.1016/j.foodres.2009.07.020

Usha Junior, R., Lakshmi, M., \& Ranjani, M. (2010). Nutritional, sensory and physical analysis of pumpkin flour incorporated into weaning mix. Malaysian Journal of Nutrition, 16(3), 379-387. PMid:22691991. 